\title{
Métodos baseados em léxico para extração de aspectos de opiniões em português
}

\author{
Raul Wagner Martins Costa e Thiago Alexandre Salgueiro Pardo
}

Núcleo Interinstitucional de Linguística Computacional (NILC)

Instituto de Ciências Matemáticas e de Computação, Universidade de São Paulo (USP)

São Carlos/SP, Brasil

raul.wagner@live.com, taspardodicmc.usp.br

\begin{abstract}
In the sentiment analysis area, the task of aspect extraction usually consists of identifying the opinion aspects in user-generated reviews of products that users have acquired. It is a basic task for several others, from polarity classification to opinion summarization. In this article, we study some methods for extraction of aspects from opinions written in Portuguese. In particular, we focus on lexical-based methods, aiming at assessing their potentialities and limitations.
\end{abstract}

Resumo. Na área de análise de sentimentos, a tarefa de extração de aspectos consiste usualmente em identificar os aspectos de opinião em revisões produzidas por usuários sobre produtos que adquiriram. É uma tarefa de base para diversas outras, desde classificação de polaridade à produção de sumários de opinião. Neste artigo, estudamos alguns métodos para extração de aspectos em opiniões escritas em português. Em especial, focamos em métodos baseados em léxico, buscando avaliar suas potencialidades e limitações.

\section{Introdução}

A web possibilitou compilar uma quantidade grande de opiniões de pessoas sobre diversos tópicos, desde política e saúde até serviços e produtos fornecidos por empresas e, além disso, extrair informações relevantes para análise e apoio à decisão em seus mais variados níveis (governamental, empresarial, comercial ou mesmo pessoal), visto que muitas das opiniões que temos possuem grande influência nas de outras pessoas, devido à natureza social dos humanos.

Estudar essas opiniões é a principal motivação da área conhecida por análise de sentimentos. Nessa área, objetiva-se extrair informações de revisões escritas por milhares de pessoas na web, principalmente. Formalmente, segundo Liu (2012), análise de sentimentos é a área de estudo que analisa as opiniões e sentimentos de pessoas com relação a entidades como produtos, serviços, organizações, indivíduos, tópicos e seus respectivos atributos/aspectos.

Como o conceito de opinião é bem amplo, no geral se estudam as opiniões que trazem consigo algum sentimento em relação a uma entidade específica. Produtos eletrônicos são objetos frequentes das pesquisas na área, dados os interesses de 
fabricantes e seus clientes. Na Figuras 1 e 2, é possível ver exemplos reais (com erros, inclusive) de revisões sobre um produto comprado online.

É um produto considerável, bom e indico para comprar fácil de usar recebi antes do prazo a empresa está de parabéns.

Figura 1. Exemplo de revisão sobre uma impressora

é um bom produto, porém algumas fahlas são encontradas na tela. O que gostei: o produtoo é bem legal. Possui alkgumas teclas de atalho interessantes. O que não gostei: os toques são mto ruins e os menus mto complicados.

Figura 2. Exemplo de revisão sobre um smartphone

Nos exemplos, nota-se que, apesar dos usuários terem opiniões positivas em relação aos produtos, eles fazem algumas críticas em relação a algumas características específicas. Isso nos motiva a analisar não só a opinião geral sobre os produtos, mas a opinião sobre as suas características de maneira individual. Para isso, é necessário identificar e extrair essas características, usualmente chamadas de "aspectos" ou "atributos" (Liu, 2012). Como exemplo, um conjunto de aspectos de um smartphone pode incluir "tela", "peso", "velocidade", "usabilidade", "design" e "bateria", dentre outros. Alguns aspectos são citados explicitamente nas revisões, enquanto outros apenas implicitamente. Por exemplo, ao usar o termo "fácil de usar" no primeiro exemplo, o usuário refere-se ao aspecto implícito "usabilidade". Também é interessante ver que nem todos os aspectos referem-se à entidade sendo avaliada. Na primeira revisão, por exemplo, a "velocidade de entrega" se refere à transportadora, e não ao produto em si. Pode-se perceber, portanto, que há vários desafios relacionados à identificação automática dos aspectos. Somam-se a isso as características do registro linguístico das opiniões da web, que se caracteriza como o chamado "internetês", onde se vê o uso de gírias, simplificações e emojis, além da ocorrência de diversos erros ortográficos e gramaticais.

O passo de identificação e extração de aspectos é a base para tarefas e aplicações seguintes mais sofisticadas, desde detecção da polaridade atribuída a cada aspecto até a sumarização das opiniões sobre uma entidade de interesse (veja, por exemplo, os trabalhos de Liu et al., 2005, López Condori e Pardo, 2017, e Silva e Pardo, 2019).

Iniciativas relativamente recentes na área têm estudado as questões relacionadas à ocorrência dos aspectos e métodos computacionais para sua extração a partir de opiniões escritas em língua portuguesa, visando aplicações mais sofisticadas de análise de sentimentos. Há abordagens com base em aprendizado de máquina (veja, por exemplo, Balage Filho, 2017), levantamento de padrões morfossintáticos e sintáticos (Balage Filho, 2017; Catharin e Feltrim, 2018), estudos linguísticos para agrupamento e organização ontológica dos aspectos (Vargas e Pardo, 2018a, 2018b, 2020) e uso de teorias discursivas na tarefa de extração de aspectos (Oliveira e Feltrim, 2015; Catharin e Feltrim, 2018). Neste trabalho, em particular, nosso interesse reside em métodos de extração de aspectos com base em conhecimento lexical, explorando uma frente até então não testada para o português e buscando delimitar suas potencialidades e limitações. 
Em uma primeira linha, baseada em conhecimento especializado, fazemos uso de ontologias de aspectos para identificar aspectos explícitos e implícitos. Em seguida, avaliamos um método genérico e simples de detecção de aspectos, que seleciona os substantivos de uma opinião como candidatos a aspecto. Aprofundando a investigação, usamos um método distribucional para enriquecer o processo, utilizando os conhecidos modelos de word embeddings (Mikolov et al., 2013). Essas abordagens foram escolhidas por apresentarem olhares distintos para a tarefa, mas que são bastante usados na literatura. Os métodos e suas variações são avaliados, demonstrando suas vantagens e limitações.

A seguir, na Seção 2, apresentamos os principais conceitos e trabalhos relacionados. Na Seção 3, detalhamos nossos métodos para extração de aspectos. A avaliação dos métodos é relatada na Seção 4. As considerações finais são feitas na Seção 5 .

\section{Trabalhos relacionados}

Um dos trabalhos pioneiros de análise de sentimentos em nível de aspecto foi feito por Hu e Liu (2004) para a língua inglesa. Os autores extraíram os aspectos através de duas estratégias. Primeiramente, foram extraídos os substantivos mais frequentes como sendo os aspectos. A segunda estratégia trata as sentenças que possuem palavras de sentimento, mas nenhuma palavra que indica um aspecto: neste caso, considera-se o substantivo mais próximo como aspecto. Com esses métodos, foram extraídos os aspectos no domínio de eletrônicos. O trabalho deles mostrou a eficácia das técnicas testadas, apesar de possuir algumas limitações, como não identificar aspectos implícitos.

Muitos trabalhos fizeram uso de técnicas de aprendizado de máquina na tarefa. Por exemplo, o estudo de Toh e Su (2015) se focou nos domínios de opiniões sobre laptops e restaurantes. Para extração dos aspectos, foi usada a técnica de aprendizado de máquina Conditional Random Fields (CRF). Para qualificação dos aspectos, foi usado um classificador binário para cada possível categoria encontrada, por exemplo, "preço", "atendimento", etc. Por fim, as duas técnicas foram agregadas para executar a tarefa completa. Os autores descobriram que o desempenho foi afetado pela pequena quantidade de vezes que muitos aspectos foram comentados pelos usuários. Liu et al. (2015) usaram CRF e redes neurais recorrentes para extração dos aspectos. O córpus usado foi o mesmo usado por Toh e Su (2015). Conclui-se que as redes neurais superam a performance de CRF. Galliat et al. (2018) foram além e empregaram word embeddings, usando o modelo word2vec de Mikolov et al. (2013), visando identificar aspectos de revisões sobre finanças em uma taxonomia manualmente construída. Os autores observaram bons resultados para a extração de aspectos explícitos, mas limitações para aspectos implícitos.

Especificamente para o português, apresentamos sucintamente alguns dos principais trabalhos conhecidos que serviram de base para o relatado aqui. É interessante notar que alguns deles seguem linhas apresentadas nos trabalhos citados anteriormente para o inglês. Há abordagens mais tradicionais, que fazem uso de aprendizado de máquina, e mais sofisticadas, que utilizam teorias discursivas. 
Balage Filho (2017) testou diferentes métodos de extração de aspectos. Foram testadas a extração de termos frequentes, a extração pela análise de relação sintática (ou seja, uso de templates sintáticos em que aspectos e seus modificadores são frequentemente encontrados) e técnicas de aprendizado de máquina. Esse estudo concluiu que conhecimento sintático e semântico tem um impacto significante na performance da extração. Além disso, mostrou que, em um córpus de resenha de livros, os resultados da extração de termos frequentes são um baseline difícil de superar.

Oliveira e Feltrim (2015) avaliaram o potencial da conhecida teoria discursiva de Centralização (Grosz et al., 1995) para prever a ocorrência de aspectos em comentários de notícias jornalísticas. Segundo a teoria da Centralização, é possível identificar "centros" de atenção mais salientes nas sentenças de acordo com suas funções sintáticas. Os autores utilizaram esses centros como representantes dos aspectos, mostrando que a extração de aspectos com base na teoria discursiva supera baselines mais simples baseados em classificação morfossintática e sintática. Dando continuidade ao trabalho anterior, Catharin e Feltrim (2018) compararam a teoria da Centralização com o uso de padrões morfossintáticos que caracterizam aspectos e de heurísticas sobre comentários de notícias e também resenhas de livros. Seus resultados foram bastante variados, mostrando que o desempenho em comentários de notícias tende a ser melhor.

Em uma abordagem relacionada, Vargas e Pardo (2018a, 2018b) realizaram um estudo manual sobre opiniões de três domínios diferentes - livros, câmeras digitais e smartphones - para propor uma organização ontológica de seus aspectos, produzindo "ontologias de aspectos", identificando tanto aspectos explícitos quanto implícitos (nesses casos, os chamados termos pista dos aspectos implícitos são registrados). Em um passo posterior, os autores propuseram um novo método para agrupamento de aspectos similares (por exemplo, "preço", "custo" e "valor"), utilizando, como base, relações lexicais e semânticas de naturezas variadas, como sinonímia, causalidade, construções deverbais, estrangeirismos e correferências, dentre outras. Seus resultados superaram abordagens tradicionais baseadas em menos conhecimento linguístico e resultados mais recentes com word embeddings, mostrando a eficácia da combinação de conhecimentos linguísticos. Vargas e Pardo (2020) vão além e propõem regras que também consideram a ocorrência de verbos psicológicos para a correta identificação de aspectos.

A seguir, apresentamos os métodos que exploramos neste artigo.

\section{Métodos}

Formalmente, os métodos investigados neste trabalho buscam, a partir de uma opinião fornecida, gerar uma lista de aspectos identificados, incluindo aspectos explícitos e implícitos. As opiniões processadas são revisões que usuários de sites de comércio eletrônico submetem, avaliando produtos que adquiriram, como as exibidas nas Figuras 1 e 2 .

Como citado anteriormente, este trabalho foca seus estudos em métodos de extração de aspectos com base em léxico, visando avaliar suas potencialidades e limitações. O processo geral é simples: se um termo na revisão sendo analisada constar no recurso léxico em uso, esse termo é extraído como um aspecto. Em geral, esse tipo 
de método demanda a disponibilidade de recursos lexicais e, portanto, costuma ser considerado caro. Recentemente, para o português, houve avanços na criação de recursos desse tipo (incluindo grandes córpus e ontologias), o que viabiliza tal investigação.

O primeiro método investigado realiza a extração de aspectos usando ontologias, que é um método já clássico na literatura. Ontologias são organizações conceituais sobre tipos de objetos e suas propriedades e relações em um domínio de conhecimento específico, como definido por Chandrasekaran et al. (1999). Neste trabalho, foram utilizadas as ontologias de aspectos criadas por Vargas e Pardo (2018a). Um exemplo de representação gráfica de uma parte dessas ontologias (para o domínio de smartphones) pode ser visto na Figura 3. Todas as palavras das ontologias (ignorando-se as relações entre elas) são utilizadas na busca por termos na revisão a ser processada. No caso de aspectos implícitos, usam-se os termos pista que também constam nas ontologias.

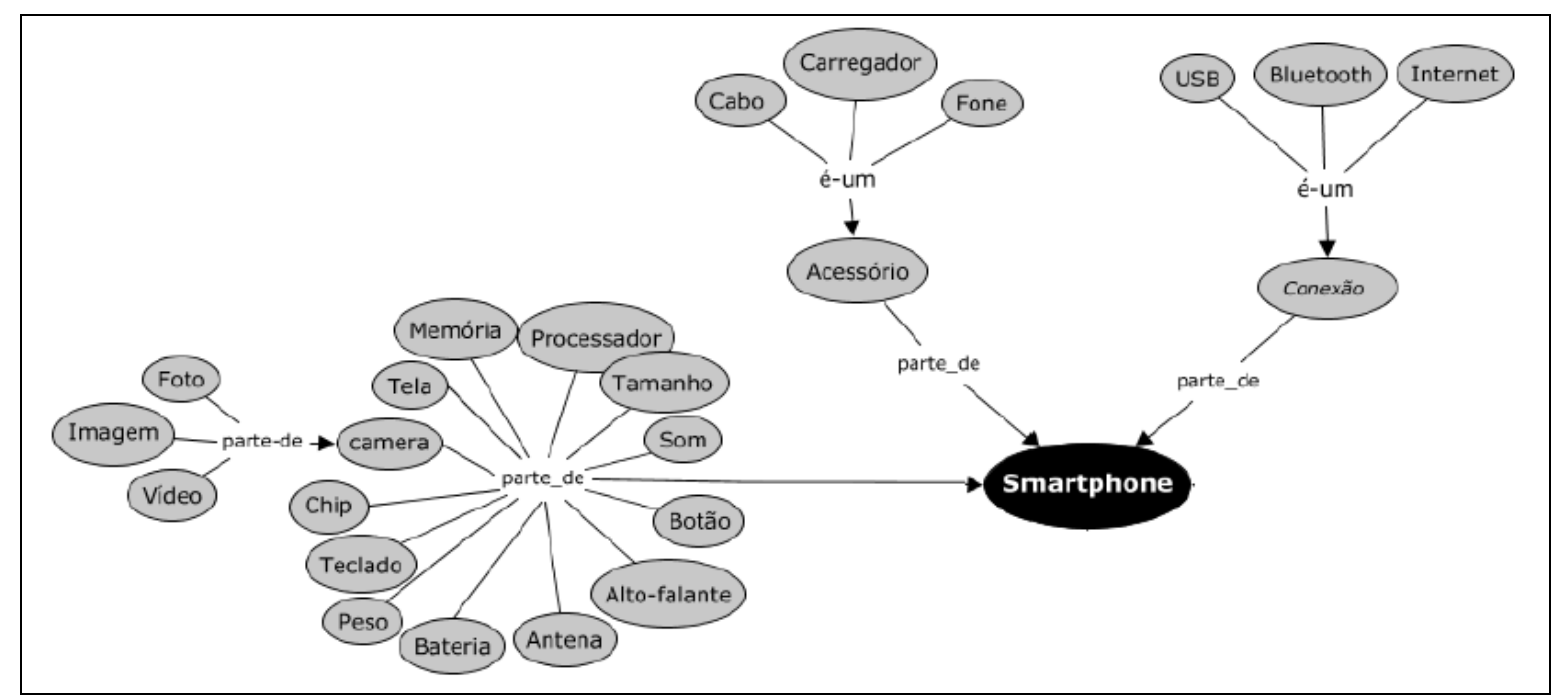

Figura 3. Exemplo de parte de ontologia de aspectos para smartphones (Vargas e Pardo, 2018a).

$\mathrm{Na}$ literatura, métodos com base em ontologias costumam atingir alta precisão, dada a qualidade dos dados ontológicos, que normalmente passam por curadoria humana. Entretanto, são métodos altamente especializados por dependerem de uma estrutura ontológica específica para um domínio de interesse.

Um segundo método avaliado, mais genérico e simples do que o anterior, usa um léxico criado com os substantivos extraídos do grande córpus de revisões de produtos eletrônicos de Hartmann et al. (2014), em uma espécie de "treinamento", para aprender os termos que podem se referir aos aspectos. Após serem extraídos desse córpus, os substantivos foram filtrados por sua frequência relativa, ou seja, de todos os substantivos recuperados, apenas os 3\% mais frequentes foram selecionados (na linha do que foi feito por Balage Filho, 2017, por exemplo). Esse valor foi visto como o melhor em testes empíricos que realizamos. Dessa forma, evita-se a inclusão de dados espúrios no processo, já que muitos substantivos não se referem a aspectos. Para extrair os substantivos, entretanto, ressalta-se que foi necessário usar o normalizador Enelvo 
(Bertaglia e Nunes, 2016) para corrigir os textos do córpus (dado que as revisões apresentam textos que não seguem necessariamente a norma culta da língua) e etiquetar morfossintaticamente cada sentença do córpus para posterior seleção dos substantivos.

Como se sabe, usar etiquetas morfossintáticas para guiar a extração de aspectos é um processo mais genérico e independente de domínio (em relação às ontologias), devendo obter uma cobertura maior de aspectos, mas sendo menos preciso, já que há muitos substantivos que não são aspectos. Ao restringir aos substantivos mais frequentes que ocorrem em um córpus de revisões de produtos, o processo fica mais informado. Entretanto, mesmo assim, tal abordagem não garante a identificação de aspectos implícitos, pois, diferentemente do que ocorre nas ontologias, os eventuais termos pista (mesmo que sejam substantivos frequentes no córpus) não estão associados aos aspectos que indicam.

Uma das principais limitações de se usar léxicos baseados em ontologias ou com substantivos extraídos de um córpus é a cobertura não exaustiva desses recursos, característica inerente a eles. Eles são incapazes de lidar, por exemplo, com palavras sinônimas não previstas. Como ilustração, um smartphone pode ser referenciado em uma revisão como "celular" ou "telefone"; se esses termos não estiverem presentes nas ontologias ou não tiverem sido usados com uma frequência mínima nos textos que deram origem ao léxico de substantivos, eles não serão reconhecidos posteriormente. Naturalmente, é inviável listar de maneira manual todas as possibilidades. Isso nos motivou a usar as word embeddings (como definidas por Mikolov et al., 2013) para enriquecer a listagem de aspectos a serem buscados nas revisões de interesse. As word embeddings são representações vetoriais de palavras em um espaço multidimensional, de forma que, se o treinamento for satisfatório, com um córpus suficientemente grande, palavras com sentido próximos ou relacionados tendem a se aglomerar no espaço vetorial.

Neste trabalho, treinamos um modelo de word embeddings com base no grande córpus de produtos eletrônicos de Hartmann et al. (2014) (que conta com 85.910 revisões de produtos variados, totalizando 4.097 .905 palavras) usando o modelo skip-

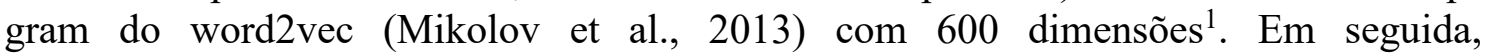
enriquecemos os recursos lexicais dos métodos anteriores com as palavras mais próximas ainda não consideradas, ou seja, para cada palavra das ontologias e do léxico de substantivo extraído do córpus, adicionamos nos recursos lexicais as 3 palavras cujos vetores na modelagem do word2vec fossem mais próximos. A proximidade dos vetores foi medida pela tradicional medida do cosseno para similaridade lexical (conforme definem Jurafsky e Martin, 2008). Dessa forma, por exemplo, para a palavra "preço", pode-se recuperar da modelagem do word2vec as palavras "valor", "custo" e "produto", que seriam as 3 mais próximas a ela no espaço vetorial treinado. Elas seriam incluídas no recurso lexical se não estivessem presentes, aumentando assim a cobertura dos recursos e, consequentemente, dos métodos.

Métodos baseados em word embeddings mostram-se bastante interessantes pelo conhecimento semântico adquirido automaticamente, mas, claramente, podem cometer

\footnotetext{
${ }^{1}$ Dentre vários modelos e dimensões testadas, essa foi a configuração de melhor desempenho. Portanto, relatamos somente os resultados desta configuração de treinamento neste artigo.
} 
erros, pois proximidade vetorial não é garantia de relação lexical relevante. Por exemplo, no caso citado acima, o termo "produto" não seria um aspecto relacionado a "preço". Na tarefa de extração de aspectos, esse tipo de associação errônea pode gerar problemas.

A Tabela 1 sintetiza o número de aspectos em cada recurso lexical criado neste trabalho. Pode-se notar que o uso das word embeddings aumentou em $47 \%$ e em $135 \%$ os recursos criados com as ontologias e os substantivos mais frequentes, respectivamente.

Tabela 1. Número de aspectos nos recursos lexicais criados

\begin{tabular}{|c|c|c|c|c|}
\hline & Ontologias & $\begin{array}{c}\text { Ontologias + } \\
\text { Word Embed. }\end{array}$ & Substantivos & $\begin{array}{c}\text { Substantivos + } \\
\text { Word Embed. }\end{array}$ \\
\hline Número de aspectos & 294 & 432 & 320 & 752 \\
\hline
\end{tabular}

A seguir, relatamos a avaliação dos métodos.

\section{Avaliação}

Foram realizados testes em um córpus anotado manualmente, composto por 105 revisões de produtos eletrônicos, em particular, câmeras digitais e smartphones, totalizando 1.980 palavras. Esse córpus é um compilado de revisões do córpus de Hartmann et al. (2014), já citado, com seus aspectos e polaridades manualmente identificados. Um exemplo de revisão que foi anotada é dado na Figura 4.

É possível observar como a anotação dos aspectos foi feita. Primeiramente, a revisão foi segmentada em orações; depois, o principal aspecto da sentença foi identificado. Por exemplo, na oração "contudo a memoria ram poderia ser $3 \mathrm{gb}$ ", "ARMAZENAMENTO" indica o grupo ao qual o aspecto extraído pertence e "memória ram" é o aspecto desejado. Pode-se ver também que, nesse caso, o aspecto foi avaliado negativamente (indicado pelo sinal '-' associado ao aspecto). 


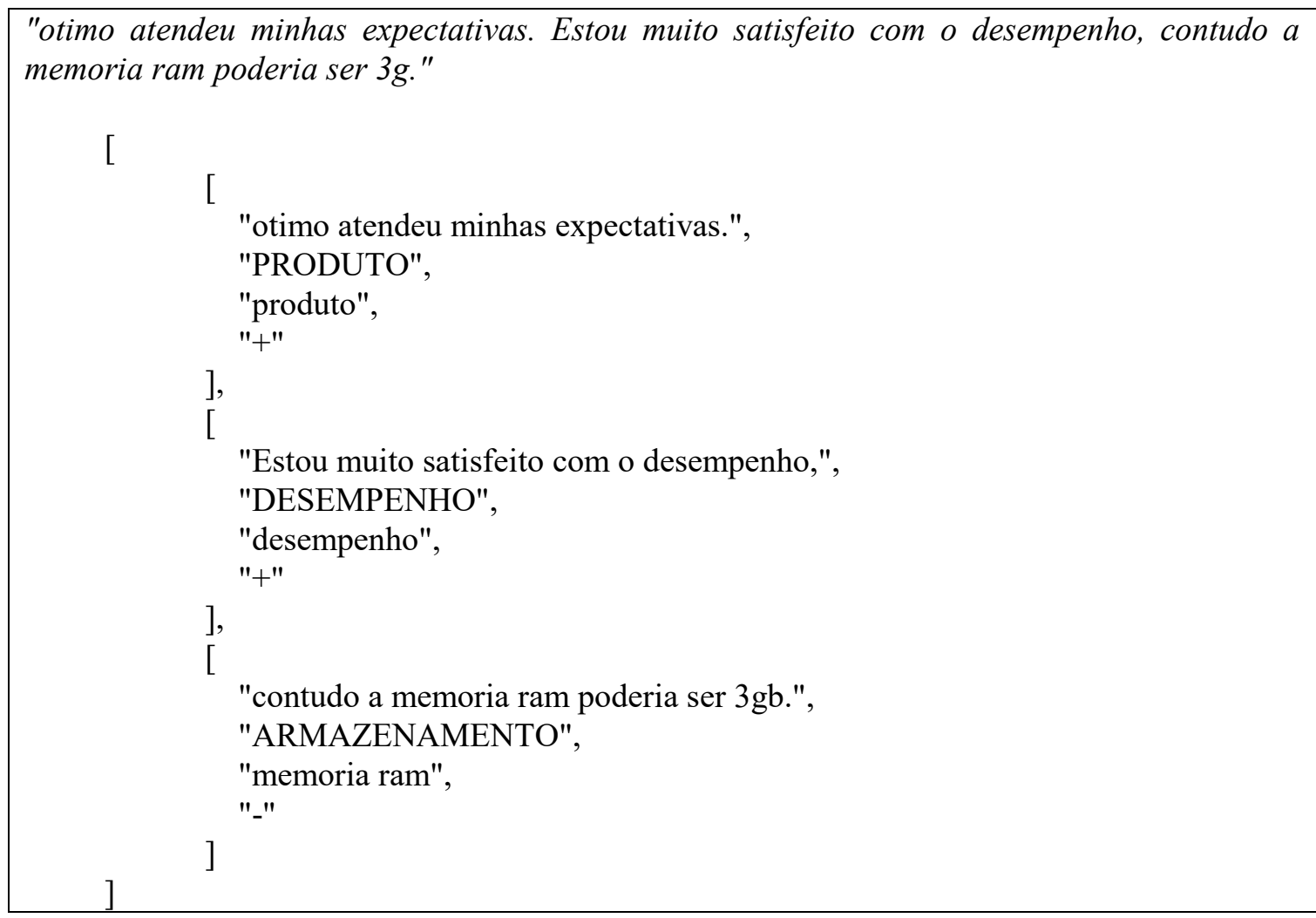

Figura 4. Exemplo de revisão anotada no córpus de teste

Para análise dos resultados dos métodos, foram escolhidas as métricas tradicionais de precisão, cobertura e medida-f, bastante comuns na área de análise de sentimentos. Precisão indica quantos aspectos extraídos estavam corretos em relação a todos os extraídos; cobertura indica quantos aspectos extraídos estavam corretos em relação a todos que deveriam ser extraídos (conforme anotação manual). A medida-f é a média harmônica de precisão e cobertura.

Para se ter uma visão mais detalhada sobre os métodos que estão sendo avaliados, foram propostos dois testes distintos, um pessimista e um otimista. A avaliação pessimista considera que um aspecto foi corretamente identificado se e somente se o aspecto extraído for exatamente igual ao aspecto anotado manualmente. Por exemplo, se o aspecto extraído for "custo" e o anotado manualmente no córpus de teste for "custo/benefício", considera-se que o aspecto não foi extraído corretamente. Já a avaliação otimista considera o exemplo anterior como um acerto, dado que "custo" está presente no termo "custo/benefício".

As médias dos resultados de cada método são exibidas na Tabela 2, sendo que os melhores resultados são mostrados em negrito. 
Tabela 2. Precisão, cobertura e medida-f obtidas pelos métodos

\begin{tabular}{|l|c|c|c|c|}
\hline \multirow{2}{*}{ Medidas } & \multicolumn{4}{|c|}{ Métodos } \\
\cline { 2 - 5 } & Ontologias & $\begin{array}{c}\text { Ontologias }+ \\
\text { Word Embed. }\end{array}$ & Substantivos & $\begin{array}{c}\text { Substantivos + } \\
\text { Word Embed. }\end{array}$ \\
\hline Precisão otimista & $\mathbf{0 , 5 3 0}$ & 0,346 & 0,150 & 0,115 \\
\hline Precisão pessimista & $\mathbf{0 , 5 2 2}$ & 0,343 & 0,150 & 0,113 \\
\hline Cobertura otimista & 0,448 & 0,433 & 0,401 & $\mathbf{0 , 4 5 8}$ \\
\hline Cobertura pessimista & $\mathbf{0 , 4 1 4}$ & 0,399 & 0,360 & 0,405 \\
\hline Medida-f otimista & $\mathbf{0 , 4 8 5}$ & 0,384 & 0,218 & 0,183 \\
\hline Medida-f pessimista & $\mathbf{0 , 4 6 1}$ & 0,368 & 0,211 & 0,176 \\
\hline
\end{tabular}

Inicialmente, é interessante notar que, como esperado e discutido na seção anterior, o método com base em ontologia obtém precisão maior do que cobertura, enquanto o com base em substantivos tem comportamento oposto. As word embeddings aumentaram a cobertura do método com base em substantivos, mas não tiveram o mesmo efeito no método com base em ontologias.

No geral, vê-se que, em termos de medida-f, o método baseado em ontologias teve um desempenho melhor, tanto em sua versão pessimista como na otimista. Esse resultado pode ser explicado pelo alto refinamento das ontologias produzidas por Vargas e Pardo (2018a) e que foram utilizadas aqui. Logicamente, pode-se esperar que a qualidade do método baseado em ontologia é diretamente influenciada pela qualidade da ontologia utilizada. Mais do que isso, depende da existência da ontologia apropriada para o domínio que analisam. Vargas e Pardo disponibilizaram ontologias apenas para os domínios de câmeras digitais, smartphones e livros, mas o portal de ontologias OntoLP ${ }^{2}$ provê outras que podem sustentar o uso do método com base em ontologias.

A análise dos erros cometidos pelos métodos evidenciou três problemas principais que afetam seus resultados. Por um lado, a vagueza nas revisões afetou o desempenho dos métodos. No córpus de teste, não é raro encontrar revisões como as mostradas na Figura 4, em que não há citação ao aspecto. Por outro lado, há aspectos muito específicos que não constavam nas ontologias ou no léxico de substantivos, e sequer foram aprendidos com a modelagem das word embeddings, como foi o caso dos aspectos "gestos" e "leitor de digitais". Além disso, a detecção de aspectos implícitos ainda é desafiadora. Apesar de as ontologias conterem os termos pista, a variedade de formas de referenciar os aspectos é muito grande.

"Bom. Quase nao utilizei ainda."
“otimo. Excelente."
"Excelente. Excelente."

Figura 4. Exemplos de revisões vagas

\footnotetext{
${ }^{2}$ http://ontolp.inf.pucrs.br/
} 
A seguir, apresentamos algumas considerações finais.

\section{Considerações finais}

Investigamos, neste artigo, métodos baseados em conhecimento lexical para a identificação e extração de aspectos. Os resultados foram promissores e o uso de ontologias se mostrou uma melhor opção em relação aos outros métodos avaliados. Entretanto, ainda há questões de pesquisa relevantes a responder em trabalhos futuros, que incluem a eficácia dos métodos em domínios variados (que, como a literatura já mostrou, é um grande problema na área) e como identificar com maior acurácia os aspectos implícitos. Futuramente, também se pretende investigar os modelos mais recentes de word embeddings contextuais, como o BERT ${ }^{3}$ (Devlin et al., 2019), que, acredita-se, pode trazer impactos mais positivos.

Mais detalhes sobre este trabalho (incluindo os dados e os softwares produzidos) e iniciativas relacionadas podem ser encontrados no site do projeto OPINANDO (https://sites.google.com/icmc.usp.br/opinando/).

\section{Agradecimentos}

Agradecemos à Pró-Reitoria de Pesquisa da Universidade de São Paulo pelo apoio a este projeto (PRP \#668).

\section{Referências}

Balage Filho, P.P. (2017). Aspect Extraction in Sentiment Analysis for Portuguese. Tese de Doutorado. Instituto de Ciências Matemáticas e de Computação, Universidade de São Paulo. São Carlos-SP, October, 77p.

Bertaglia, T.F.C. and Nunes, M.G.V. (2016). Exploring Word Embeddings for Unsupervised Textual User-Generated Content Normalization. In the Proceedings of the 2nd Workshop on Noisy User-generated Text (WNUT), pp. 112-120.

Catharin, L.G. and Feltrim, V.D. (2018). Finding Opinion Targets in News Comments and Book Reviews. In the Proceedings of the 13th International Conference on Computational Processing of the Portuguese Language (PROPOR) (LNAI 11122), pp. 375-384.

Chandrasekaran, B.; Josephson, J.; Benjamins, V. R. (1999). What Are Ontologies, and Why Do We Need Them? IEEE Intelligent Systems and their Applications, Vol. 14, N. 1, pp. 20-26.

Devlin, J.; Chang, M.W.; Lee, K.; Toutanova, K. (2019). BERT: Pre-training of Deep Bidirectional Transformers for Language Understanding. In the Proceedings of the Annual Conference of the North American Chapter of the Association for Computational Linguistics: Human Language Technologies (NAACL-HLT), pp. 4171-4186.

\footnotetext{
3 Já treinado e disponibilizado para a língua portuguesa (Souza et al., 2020), como se pode ver em https://github.com/neuralmind-ai/portuguese-bert
} 
Gaillat, T.; Stearns, B.; Sridhar, G; McDermott, R.; Zarrouk, M. (2018). Implicit and Explicit Aspect Extraction in Financial Microblogs. In the Proceedings of the 1st Workshop on Economics and Natural Language Processing, pp. 55-61.

Grosz, B.J.; Joshi, A.K.; Weinstein, S. (1995). Centering: A Framework for Modeling the Local Coherence of Discourse. Computational Linguistics, Vol. 21, N. 2, pp. 204225.

$\mathrm{Hu}, \mathrm{M}$. and Liu, B. (2004). Mining and summarizing customer reviews. In the Proceedings of the tenth ACM SIGKDD International Conference on Knowledge Discovery and Data Mining (KDD), pp. 168-177.

Hartmann, N.S.; Avanço, L.V.; Balage Filho, P.P.; Duran, M.S.; Nunes, M.G.V.; Pardo, T.A.S.; Aluísio, S.M. (2014). A Large Corpus of Product Reviews in Portuguese: Tackling Out-Of-Vocabulary Words. In the Proceedings of the 9th Language Resources and Evaluation Conference (LREC), pp. 3865-3871.

Jurafsky, D. and Martin, J.H. (2008). Speech and Language Processing. An Introduction to Natural Language Processing, Computational Linguistics, and Speech Recognition. Prentice Hall.

Liu, B. (2012). Sentiment Analysis and Opinion Mining. Morgan \& Claypool Publishers.

Liu, B; Hu, M.; Cheng, J. (2005). Opinion Observer: Analyzing and Comparing Opinions on the Web. In the Proceedings of the 14th International Conference on World Wide Web, pp. 342-351.

Liu, P.; Joty, S.; Meng, H. (2015). Fine-grained Opinion Mining with Recurrent Neural Networks and Word Embeddings. In the Proceedings of the Conference on Empirical Methods in Natural Language Processing (EMNLP), pp. 1433-1443.

López Condori, R.E. and Pardo, T.A.S. (2017). Opinion Summarization Methods: Comparing and Extending Extractive and Abstractive Approaches. Expert Systems with Applications (ESWA), Vol. 78, pp. 124-134.

Mikolov, T.; Corrado, G.; Chen, K.; Dean, J. (2013). Efficient Estimation of Word Representations in Vector Space. ArXiv: 1301.3781.

Oliveira, F.W.C. and Feltrim, V.D. (2015). Extração de Alvos em Comentários de Notícias em Português baseada na Teoria da Centralização. In the Proceedings of Symposium in Information and Human Language Technology (STIL), pp. 63-67.

Silva, R.R. e Pardo, T.A.S. (2019). Córpus 4P: um córpus anotado de opiniões em português sobre produtos eletrônicos para fins de sumarização contrastiva de opinião. In Anais da 6a Jornada de Descrição do Português (JDP), pp. 330-338.

Souza, F.; Nogueira, R.; Lotufo, R. (2020). Portuguese Named Entity Recognition using BERT-CRF. arXiv:1909.10649v2

Toh, Z. and Su, J. (2015). NLANGP: Supervised Machine Learning System for Aspect Category Classification and Opinion Target Extraction. In the Proceedings of the 9th International Workshop on Semantic Evaluation (SemEval), pp. 496-501. 
Vargas, F.A. and Pardo, T,A.S. (2018a). Hierarchical clustering of aspects for opinion mining: a corpus study. In M.J.B. Finatto, R.R. Rebechi, S. Sarmento and A.E.P. Bocorny (eds.), Linguística de Corpus: Perspectivas, pp. 69-91. Porto Alegre: Instituto de Letras da UFRGS. 580p.

Vargas, F.A. and Pardo, T.A.S. (2018b). Aspect clustering methods for sentiment analysis. In the Proceedings of the 13th International Conference on the Computational Processing of Portuguese (PROPOR) (LNAI 11122), pp. 365-374.

Vargas, F.A. and Pardo, T.A.S. (2020). Linguistic Rules for Fine-Grained Opinion Extraction. In the Workshop Proceedings of the 14th International AAAI Conference on Web and Social Media, pp. 1-6. 